are either roughly constant or increase with depth throughout the upper mantle below the asthenosphere $^{14-16}$, suggesting that water content should follow similar trends. This enigma can be solved if water is not the primary cause of seismic attenuation, as shown by Cline and colleagues.

Nevertheless, there are some issues regarding the applicability of Cline and colleagues' results to the actual mantle. For instance, the authors artificially increased the water content of some of their olivine aggregates using a technique called doping, in which a trace amount of one element is substituted for another. This process introduced artificial crystal defects whose mobility might differ from the defects intrinsic to olivine - although the authors argue that these artificial defects do not affect their conclusions. The effect of oxygen fugacity on the mobility of these different types of defect is also unknown.

Future studies on the seismic properties of olivine could avoid the need for doping by subjecting aggregates to higher pressures than those used by Cline and colleagues. For example, measurements could be made using an oscillation technique that combines a largevolume press and X-ray observations ${ }^{17}$. Future experiments should include wider ranges of oxidation conditions and olivine grain sizes than those considered by Cline et al., to confirm the dominance of oxygen fugacity over other causes of anelastic behaviour.

Although some petrological evidence suggests that oxygen fugacity in the mantle generally decreases with depth ${ }^{12,13}$, it has been difficult to evaluate how such oxidation states vary laterally. The probable link between oxygen fugacity and attenuation of seismic waves in peridotite could enable 3D mapping of oxidation states in the deep mantle, using data obtained with an imaging technique called seismic tomography. Meanwhile, the lack of correlation between water content in mantle olivine and seismic attenuation, if confirmed by independent studies at higher pressures, might require scientists to reconsider the role of water in the softening of mantle rocks, and the distribution and circulation of water throughout the deep Earth..

Tetsuo Irifune and Tomohiro Ohuchi are in the Geodynamics Research Center, Ehime University, Matsuyama 790-8577, Japan. T.I. is also at the Earth Life Science Institute, Tokyo Institute of Technology. e-mails: irifune@dpc.ehime-u.ac.jp; ohuchi@sci.ehime-u.ac.jp

1. Dalton, C. A., Ekström, G. \& Dziewoński, A. M. J. Geophys. Res. Solid Earth 113, B09303 (2008).

2. Cline, C. J. II, Faul, U. H., David, E. C., Berry, A. J. \& Jackson, I. Nature 555, 355-358 (2018).

3. Anderson, D. L. \& Spetzler, H. Phys. Earth Planet. Inter. 4, 62-64 (1970)

4. Wilcock, W. S. D., Solomon, S. C., Purdy, G. M. \& Toomey, D. R. Science 258, 1470-1474 (1992)

5. Hirth, G. \& Kohlstedt, D. L. J. Geophys. Res. Solid Earth 100, 15441-15449 (1995).

6. Karato, S. \& Jung, H. Earth Planet. Sci. Lett. $\mathbf{1 5 7}$ 193-207 (1998).

7. McDonnell, R. D., Peach, C. J. \& Spiers, C. J. J. Geophys. Res. Solid Earth 104, 17823-17845 (1999).

8. Ohuchi, T. et al. Sci. Adv. 1, e1500360 (2015).

9. Karato, S., Paterson, M. S. \& FitzGerald, J. D. J. Geophys. Res. Solid Earth 91, 8151-8176 (1986) 10. Hirschmann, M. M. Annu. Rev. Earth Planet. Sci. 34, 629-653 (2006).

11.Jackson, I. \& Paterson, M. S. Pure Appl. Geophys. 141, 445-466 (1993)

12. McCammon, C. \& Kopylova, M. G. Contrib. Mineral. Petrol. 148, 55-68 (2004).

13. Woodland, A. B. \& Koch, M. Earth Planet. Sci. Lett. 214, 295-310 (2003).

14.Sarafian, E. et al. Geochem. Geophys. Geosyst. 16 , 1115-1132 (2015).

15.Utada, H., Koyama, T., Obayashi, M. \& Fukao, Y. Earth Planet. Sci. Lett. 281, 249-257 (2009)

16. Yoshino, T., Matsuzaki, T., Yamashita, S. \& Katsura, T. Nature 443, 973-976 (2006).

17.Li, L. \& Weidner, D. J. Rev. Sci. Instrum. 78, 053902 (2007).

tissue ${ }^{5}$, and a third used radiocarbon dating to identify hippocampal-neuron turnover ${ }^{6}$. However, methodological challenges make human studies difficult to interpret, and more are required to make definitive conclusions.

Sorrells et al. set out to address this need using classic immunohistochemical techniques in which specific antibodies are bound to proteins of interest, revealing their locations in tissue. The authors used this strategy to count neural precursor cells, proliferating cells and immature neurons in samples from 59 human subjects, spanning fetal development through to old age (Fig. 1). They found streams of all three cell types migrating from an embryonic 'germinal zone' to the developing dentate gyrus at 14 weeks of gestation. By 22 weeks, migration was reduced, and immature neurons were largely restricted to the dentate gyrus. And there were many fewer immature neurons at one year of life than at earlier stages. The oldest sample containing immature neurons was taken from a 13-year-old individual. These findings are in stark contrast to the prevailing view that human hippocampal neurogenesis extends throughout adult life.

Is it possible to reconcile the findings with previous human data? Although direct comparisons are difficult, Sorrells et al. offer some explanations. For example, they find that DCX and PSA-NCAM, two proteins that reliably mark immature neurons in animals, can label mature neurons and non-neuronal glial cells in humans. Indeed, the authors show that these two markers unambiguously identify immature neurons only if both are expressed in a demands. But it was not always so. In the 1960s, biologist Joseph Altman reported 


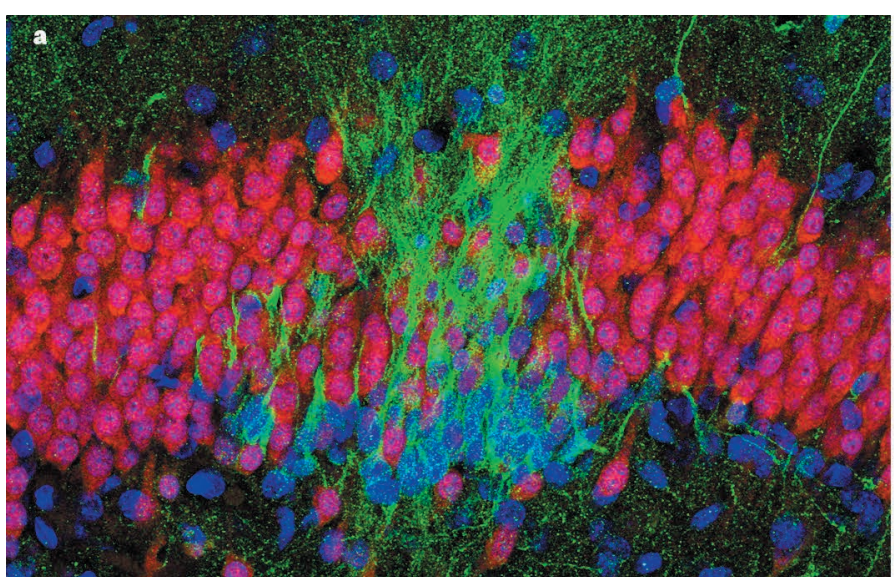

Figure 1 | Decreasing neurogenesis with age. Sorrells et al. ${ }^{1}$ examined slices of the hippocampus from human brains at various stages of life, to investigate when new neurons are generated. Green indicates the location of the protein DCX, which is produced in new neurons; red indicates the protein NeuN,

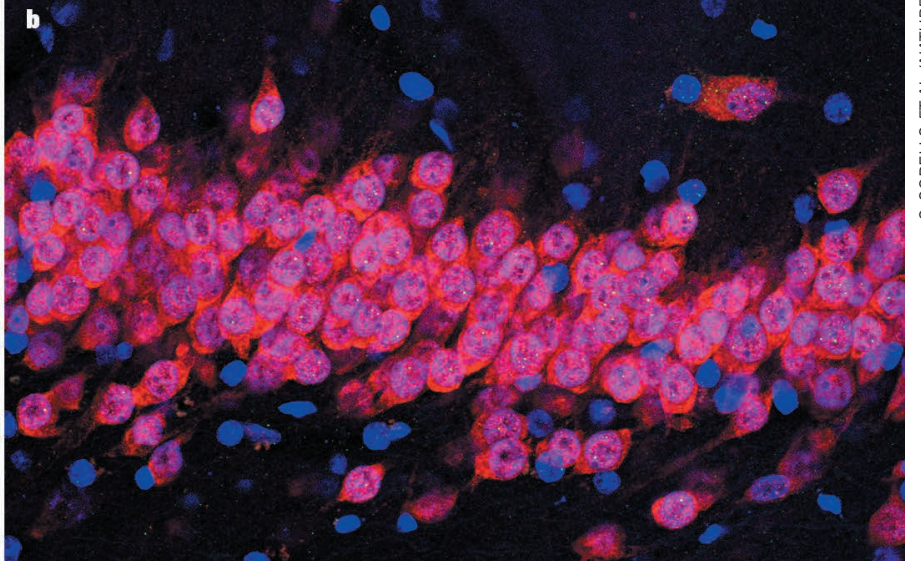

which is produced in mature neurons; blue indicates a fluorescent marker called DAPI, which stains all cell nuclei. a, At birth, many new neurons can be seen. $\mathbf{b}$, By contrast, the authors observed no new neurons in the adult hippocampus. cell. Similarly, the group demonstrated that it is possible to obtain BrdU-like immunohistochemical labelling in tissue that did not actually contain BrdU. Nonspecific labelling could therefore have led to false-positive results in previous studies.

The researchers' careful approach also speaks to the challenges of performing neurogenesis work in humans. Animal studies have shown that PSA-NCAM is modified by previous experiences ${ }^{7}$ and that DCX degrades if tissue is not rapidly preserved ${ }^{8}$. An apparent loss of neurogenesis could therefore reflect changes in marker expression, especially if stringent criteria are used to define new neurons. Given that there are debates about hippocampal precursor-cell identity even in rodents ${ }^{9}$, it is also possible that we simply do not know what to look for in humans.

Sorrells et al. minimized these issues in several ways. First, they observed neurogenesis in the hippocampus of infants and children, which served as a positive control. Second, they used a variety of adult samples to minimize the possibility that problems with tissue health or preservation could confound their results. Third, they used diverse markers of neurogenesis to gain multiple lines of evidence. Nonetheless, further investigation will be needed to see whether Sorrells and colleagues' conclusions will stand the test of time.

How do the authors' findings fit with the animal literature? With a bit of conceptual recalibration, they might fit quite well. Rodents are born with relatively immature nervous systems, so adult rodent neurogenesis could be a decent model of neurogenesis in children or adolescents. Given that depression, schizophrenia and Alzheimer's disease are rooted in early hippocampal defects, even neurons generated in childhood could have a key role in the aetiology of disease in humans. In addition, primate data ${ }^{10}$ suggest that new neurons in humans could go through an extended period of maturation (years or even decades) relative to what occurs in rodents, during which time they might have enhanced plasticity and important functional properties. Thus, whereas the continual addition of new neurons might provide plasticity in adult rodents, the prolonged development of neurons could provide a similar plasticity in adult humans.

At the other end of the developmental spectrum, even in rodents, neurogenesis is very low by middle age 2 . Thus, Sorrells and colleagues' human data again are not wholly inconsistent with the animal literature. If the focus of rodent studies were shifted to identifying the mechanisms by which neurogenesis diminishes over time, and to how neurogenesis can be enhanced to offset pathology caused by age and disease, we just might be able to translate the authors' sobering findings into discoveries that improve human health. -
Jason S. Snyder is in the Department of Psychology, Djavad Mowafaghian Centre for Brain Health, University of British Columbia, Vancouver, British Columbia V6T 1Z3, Canada. e-mail:jasonsnyder@psych.ubc.ca

1. Sorrells, S. F. et al. Nature 555, 377-381 (2018).

2. Altman, J. \& Das, G. D. J. Comp. Neurol. 124, 319-335 (1965).

3. Gross, C. G. Nature Rev. Neurosci. 1, 67-73 (2000)

4. Eriksson, P. S. et al. Nature Med. 4, 1313-1317 (1998)

5. Knoth, R. et al. PLoS ONE 5, e8809 (2010).

6. Spalding, K. L. et al. Cell 153, 1219-1227 (2013).

7. Lopez-Fernandez, M. A. et al. J. Neurosci. 27, 4552-4561 (2007).

8. Boekhoorn, K., Joels, M. \& Lucassen, P. J. Neurobiol. Dis. 24, 1-14 (2006).

9. Bonaguidi, M. A. et al. Cold Spring Harb. Perspect. Biol. 8, a018838 (2016)

10. Kohler, S. J., Williams, N. I., Stanton, G. B., Cameron, J. L. \& Greenough, W. T. Proc. Natl Acad. Sci. USA 108, 10326-10331 (2011).

This article was published online on 7 March 2018.

\section{Landscapes of childhood tumours}

\section{Two analyses of the genetic alterations that characterize paediatric cancers reveal key differences from adult cancers, and point to ways of optimizing therapeutic approaches to combating cancer in children. SEE ARTICLE P.321 \& LETTER P.371}

\section{PRATITI BANDOPADHAYAY \& MATTHEW MEYERSON}

I The mapping of the human genome, followed by the explosion in nextgeneration genome sequencing, has revolutionized our understanding of cancer. These advances have paved the way for precision-medicine approaches to treating adult cancers. Two papers in Nature report the first pan-cancer genomic analyses in children. In the first, Gröbner et al. ${ }^{1}$ (page 321) analysed sequences of whole exomes (all the proteincoding regions in the genome) or whole genomes for 961 cancers across 24 tumour types, with an emphasis on tumours of the central nervous system. In the second, $\mathrm{Ma}$ et $a .^{2}$ (page 371 ) used similar analyses to characterize 1,699 cancers across 6 types of cancer tissue, particularly leukaemias. 